https://helda.helsinki.fi

\title{
Transaortic Transcatheter Aortic Valve Implantation as a second choice over the Transapical access
}

Ropponen, J.

2016-03

Ropponen , J , Vainikka , T , Sinisalo , J , Rapola , J , Laine , M \& Ihlberg , L 2016 , '

Transaortic Transcatheter Aortic Valve Implantation as a second choice over the Transapical

access ' , Scandinavian Journal of Surgery , vol. 105 , no. 1 , pp. 35-41 . https://doi.org/10.1177/1457496915575832

http://hdl.handle.net/10138/161330

https://doi.org/10.1177/1457496915575832

publishedVersion

Downloaded from Helda, University of Helsinki institutional repository.

This is an electronic reprint of the original article.

This reprint may differ from the original in pagination and typographic detail.

Please cite the original version. 


\title{
TRANSAORTIC TRANSCATHETER AORTIC VALVE IMPLANTATION AS A SECOND CHOICE OVER THE TRANSAPICAL ACCESS
}

\author{
J. Ropponen ${ }^{1}$, T. Vainikka', J. Sinisalo ${ }^{2}$, J. Rapola ${ }^{2}$, M. Laine ${ }^{2}$, L. Ihlberg1 \\ ${ }^{1}$ Department of Cardiac Surgery, Heart and Lung Center, Helsinki University Hospital, Helsinki, Finland \\ 2 Department of Cardiology, Heart and Lung Center, Helsinki University Hospital, Helsinki, Finland
}

\begin{abstract}
Background and Aims: In this report, we present our experience with the transaortic transcatheter aortic valve implantation using the SAPIEN valve. The procedural success, 30 -day outcome, and survival up to 2 years are compared with the transapical access performed in patients in our institution.

Material and Methods: Of a total of 282 transcatheter aortic valve implantation patients, 100 consecutive patients had a non-transfemoral approach. The transaortic and transapical access routes were used in 36 and 64 patients, respectively. The transaortic group had a higher mean logistic EuroSCORE (32.6 vs $25.2, p=0.021)$ and more patients with left ventricular ejection fraction less than $40 \%(33.3 \%$ vs $14.1 \%, p=0.023)$.

Results: The respective technical success rates for the transaortic and transapical groups were $100 \%$ and $95.2 \%(p=N S)$. There were significantly more perioperative hemodynamic problems necessitating cardiopulmonary resuscitation or mechanical circulatory support in the transapical group $(18.8 \%$ vs $2.8 \%, p=0.023)$. The transaortic group had a slightly shorter hospital stay ( 7 vs 8 days, $p=0.018$ ). The 30 -day mortality was $8.6 \%$ and $10.9 \%$ in the transaortic and transapical group, respectively $(\mathrm{p}=\mathrm{NS})$. Combined safety outcome was similar in both groups at 30 days. The respective 1-year survival rates for the transaortic and transapical groups were $71.5 \%$ and $68.3 \%$, respectively $(p=N S)$.

Conclusion: The trans transcatheter aortic valve implantation is a considerable choice to transapical approach. Despite a higher risk patient cohort, the clinical outcome is at least comparable to the transapical transcatheter aortic valve implantation, and it can be utilized as a second choice for patients with prohibitive iliac-femoral anatomy for transfemoral access.
\end{abstract}

Key words: Heart diseases; aortic valve stenosis; heart surgical procedure; cardiac valve prosthesis; transcatheter aortic valve implantation; minimally invasive surgical procedures

Correspondence:

Leo Ihlberg, M.D., Ph.D.

Department of Cardiac Surgery

Heart and Lung Center

Helsinki University Hospital

P.O. Box 340

FI-00029 Helsinki

Finland

Email: leo.ihlberg@hus.fi

Scandinavian Journal of Surgery

2016, Vol. 105(1) 35-41

(c) The Finnish Surgical Society 2015

Reprints and permissions:

sagepub.co.uk/journalsPermissions.nav

DOI: $10.1177 / 1457496915575832$

sjs.sagepub.com

@SAGE 


\section{INTRODUCTION}

Over the recent years, transcatheter aortic valve implantation (TAVI) has evolved from an experimental treatment modality to an essential part of clinical practice for patients with symptomatic severe aortic stenosis (AS) and who have either a high or prohibitive risk for an open surgical aortic valve replacement (AVR) (1). In the majority of institutes, the transfemo$\mathrm{ral}(\mathrm{TF})$ is the preferred access route whenever it is feasible. Even with this policy, an alternative access route is required in up to $30 \%$ of patients (2). Several different options have been introduced, but for the SAPIEN valve, the transapical (TA) route is the original and most widely used alternative for the TF. However, the TA approach is reported to have a higher acute complication rate and inferior survival than the TF (3-5). The novel transaortic (TAo) approach has recently been introduced as an alternative option for the TA (6, $7)$. The TAo has potential theoretical advantages over the TA $(6,7)$, but up-to-date limited comparable data have been published on this matter $(7,8)$.

In this report, we present our experience with the TAo-TAVI using the SAPIEN valve. The procedural success, 30-day outcome, and survival up to 2 years are compared with our institutional TA patient cohort.

\section{MATERIAL AND METHODS}

Between December 2008 and September 2014, 282 consecutive inoperable or high-risk patients with severe symptomatic AS were treated with the TAVI at the Helsinki University Hospital, Finland. These patients were prospectively included in our TAVI database. Of these, 100 patients who had a non-TF approach were included in this study. Adhering to the European Society of Cardiology (ESC)/European Association for Cardio-Thoracic Surgery (EACTS) treatment guidelines (9), the patients were considered for TAVI if they were deemed to be in high or prohibitive risk for the open surgical AVR. The EuroSCORE risk calculator was used as an aid in patient selection. The decision to proceed with TAVI was discussed by a dedicated heart team including experienced clinical and interventional cardiologists, cardiovascular surgeons, and cardiac anesthetists. All selected patients underwent complete clinical work-up that comprised physical examination, baseline laboratory testing, and evaluation of pulmonary function and echo-Doppler study of the carotid arteries. The cardiac studies included a transthoracic echocardiography (TTE) and/or transesophageal echocardiography (TEE) and a coronary angiogram. For the technical feasibility of the TAVI, the gated multidetector computed tomography (MDCT) has become the primary imaging study to assess the anatomy of the aortic valve, aortic root, valve sizing, and vasculature with respect to the access options. Patients were selected to undergo the TAVI through the TF route whenever it was not prohibited by the size, calcification, or tortuosity of the iliac-femoral arteries. From 2008 to June 2011, the TA approach was utilized as the only alternative option to the TF-TAVI. Thereafter, the choice between the TAo and TA has been performed by the discretion of the heart team, being first utilized to the extreme-risk TAVI patients, such as those deemed to have a hostile apex (extreme friability, morbid obesity, or high-dose immunosuppressive medication, etc.), poor left ventricular function, or severe chronic obstructive pulmonary disease (COPD). After the technical feasibility of the TAo-TAVI was established, it has been increasingly utilized as a second choice default option over the TA access.

The procedures were performed under general anesthesia using both fluoroscopic and TEE guidance using Edwards SAPIEN Transcatheter Heart Valve (Edwards Lifesciences, Irvine, CA). Over the course of time, all different generation SAPIEN valves (SAPIEN, XT, and S3) were used. The TAo-TAVI and TA-TAVI were performed as previously described $(2,7)$.

With respect to the TAo-TAVI, the access for all cases to the distal ascending aorta was gained via upper partial sternotomy that was J-shaped to the right second or third intercostal space. Two pledgeted purse-string sutures were placed at the selected spot, which was chosen both by identifying a calcific-free spot in the preoperative MDCT and by directly palpating the aorta at a minimum distance of $5 \mathrm{~cm}$ from the aortic annulus. A direct needle puncture was performed to introduce a soft wire and a $6 \mathrm{~F}$ multipurpose (MPA) catheter (Boston Scientific Inc., Natick, MA). After the aortic valve was crossed with the wire, a pre-shaped ExtraStiff Amplatz guide wire (Boston Scientific Corp., Natick, MA) was placed into the left ventricle followed by insertion of the Ascendra ${ }^{\circledR}$ sheath into the ascending aorta. First, a balloon valvuloplasty was performed with a $20 \mathrm{~mm} \times 30 \mathrm{~mm}$ balloon followed by an Edwards SAPIEN valve deployment into the desired position. Both valvuloplasty and valve implantation were performed during short bursts of rapid ventricular pacing at $160-180$ beats / $\mathrm{min}$.

The main study outcomes were Valve Academic Research Consortium (VARC)-defined procedural technical success, 30-day combined safety endpoint, and 12-month survival. According to VARC criteria, the technical success is defined as gaining successful vascular access, successful delivery and deployment of a single valve at the correct anatomical position, and appropriate prosthetic valve function without significant (moderate or severe) aortic regurgitation (AR) or stenosis, as assessed by echocardiography immediately after valve deployment and prior to hospital discharge (9). The combined safety outcome at 30 days is a composite of all-cause mortality, major stroke, disabling bleeding, severe (Stage 3) acute kidney injury (AKI), post-procedure myocardial infarction (MI), major vascular complication, and a repeat procedure for valve-related dysfunction (9).

The data were collected on a dedicated case report form. The pertinent demographics and patient risk factors were defined according to the criteria of the EuroSCORE registry. In addition, several intraprocedural factors were also registered, such as operative time, need for mechanical hemodynamic support or resuscitation, and blood product usage (for the first $24 \mathrm{~h})$. The length of intensive care unit (ICU) and hospital stay was recorded. After discharge, patients were followed both clinically and by TTE at 3 months and annually thereafter. 
TABLE 1

Demographics and baseline characteristics.

\begin{tabular}{llll}
\hline Variable & Transaortic (TAo) & Transapical (TA) & p value \\
\hline Female sex & $52.8(19)$ & $53.1(34)$ & 0.973 \\
Age (years) & $81.7 \pm 7.1$ & $79.4 \pm 7.7$ & 0.142 \\
Logistic EuroSCORE & $32.6 \pm 19.5$ & $25.2 \pm 12.2$ & 0.021 \\
NYHA-class & & & $9.4(6)$ \\
$\quad$ II & $2.9(1)$ & $73.4(47)$ & 0.419 \\
III & $74.3(26)$ & $17.2(11)$ & 0.023 \\
IV & $22.9(8)$ & $14.1(9)$ & 0.466 \\
LVEF <40\% & $33.3(12)$ & $70.3(45)$ & 0.153 \\
Coronary artery disease & $77.1(27)$ & $19.0(12)$ & 0.755 \\
Prior MI & $8.3(3)$ & $25.0(16)$ & 0.518 \\
CVD & $22.2(8)$ & $28.1(18)$ & \\
Diabetes & $22.2(8)$ & & 0.306 \\
Chronic renal disease & & $65.1(41)$ & 0.184 \\
Peripheral vascular disease & $75.0(27)$ & $38.1(24)$ & 0.538 \\
COPD & $25.0(9)$ & $40.1(26)$ & \\
Previous open heart surgery & $30.6(11)$ & & \\
\hline
\end{tabular}

NYHA: New York Heart Association; LVEF: left ventricular ejection fraction; MI: myocardial infarction; CVD: cerebrovascular disease; COPD: chronic obstructive pulmonary disease.

Values are given as percentage (number of patients) or mean \pm standard deviation.

Qualitative data are expressed as frequencies and percentages, and differences between groups were compared with the chi-square test or Fisher's exact test, as appropriate. Normally distributed quantitative data are expressed as mean \pm standard deviations, and skewed data are presented as median with its interquartile range (first quartile-third quartile) and analyzed with the non-parametric Mann-Whitney U-test or Student's $t$-test, as appropriate. Late survival was assessed by Kaplan-Meier analysis, and the log-rank test was used to determine the difference in mortality. Differences with a two-sided $p$ value $<0.05$ were considered as statistically significant. The SPSS version 20.1 was used for the statistical calculations (IBMCorp, Armonk, NY).

\section{RESULTS}

The patient demographics and baseline characteristics are listed in Table 1 . The mean age was $80.2 \pm 7.5$ years, and $53 \%$ of the patients were female. The TAo group presented an overall higher risk cohort as their mean logistic EuroSCORE was 32.6 compared to 25.1 in the TA group $(\mathrm{p}=0.021)$. In addition, more TAo patients had a decreased left ventricular function $(33.3 \%$ vs $14.3 \%, \mathrm{p}=0.023)$. The patient groups did not differ with respect to any other baseline characteristics.

The procedural information is depicted in Table 2. The average device size was larger in the TAo group $(p=0.019)$. The respective technical success rates for the TAo and TA groups were $100 \%$ and $95.2 \%$, respectively $(p=N S)$. There was no difference in the procedural time between the groups. The combined rate of patients who required cardiopulmonary resuscitation (CPR) and/or mechanical hemodynamic support was $2.8 \%$ in the TAo group and $18.8 \%$ in the TA group $(p=0.023)$. Three intraprocedural deaths occurred in the TA group as opposed to none in the TAo group.
These deaths were caused by hemodynamic collapse in two occasions. The first patient did not recover after rapid pacing at the valve implantation and was unresponsive to the CPR. The second had an undersized valve deployed that resulted in a severe paravalvular leak and progressive hemodynamic deterioration. The third intraprocedural death was caused by an aortic root rupture of a heavily calcified bicuspid valve on a patient who was deemed not to be a candidate for rescue open heart surgery.

Two additional patients in the TA group were lost within the first day. The first death was caused by an apical access site bleeding. The other was due to, after a seemingly uncomplicated and successful implantation, device migration to ventricular direction leading to an overhang of the native aortic valve leaflets, severe AR, and hemodynamic collapse $8 \mathrm{~h}$ post-procedure.

The 30 -day mortality was $8.6 \%$ and $10.9 \%$ in the TAo and TA group, respectively $(\mathrm{p}=\mathrm{NS})$ (Table 3$)$. There were no major strokes or myocardial infarcts in either group, whereas one patient had a Stage 3 kidney failure. Combined safety outcome was met in $11.1 \%$ and $14.1 \%$ in the TAo and TA groups, respectively $(p=N S)$

After the TAVI, the transvalvular gradients decreased to $15.6 \pm 5.4 \mathrm{mmHg}$ (peak) and $8.8 \pm 3.4 \mathrm{mmHg}$ (mean) at 3 months with no difference between the groups (Table 4). All patients had intravalvular regurgitation graded either none/trace or mild. A total of $4.1 \%$ of patients had paravalvular leakage that was seen as more than mild in its severity.

The respective 1-year survival rates for the TAo and TA groups were $71.5 \%$ and $68.3 \%$ (Fig. 1), respectively. As the survival curves are virtually overlapping and crossing each other twice, no log-rank testing for difference was performed. The causes of death within 1 year are listed in Table 5. 
TABLE 2

Perioperative data.

\begin{tabular}{|c|c|c|c|}
\hline Variable & Transaortic (TAo) & Transapical (TA) & $\mathrm{p}$ value \\
\hline \multicolumn{4}{|l|}{ Device size } \\
\hline 23 & $27.8(10)$ & $36.5(23)$ & \\
\hline 26 & $30.6(11)$ & $49.2(31)$ & \\
\hline 29 & $41.7(15)$ & $14.3(9)$ & 0.019 \\
\hline Predilation valvuloplasty & $94.4(34)$ & $82.5(52)$ & 0.092 \\
\hline Procedure time (min) & $91(80-100)$ & $83(72-95)$ & 0.257 \\
\hline Technical success ${ }^{a}$ & $100(36)$ & $93.8(60)$ & 0.126 \\
\hline Unsuccessful vascular access & $0(0)$ & $1.6(1)$ & \\
\hline Second valve implanted & $0(0)$ & $3.2(2)$ & 0.280 \\
\hline Severe paravalvular regurgitation & $0(0)$ & $1.6(1)$ & 0.419 \\
\hline Periprocedural hemodynamic collapse $\mathrm{e}^{\mathrm{b}}$ & $2.8(1)$ & $18.8(12)$ & 0.023 \\
\hline CPR & $2.8(1)$ & $14.1(9)$ & 0.071 \\
\hline IABP & $0(0)$ & $4.7(3)$ & 0.187 \\
\hline Cardiopulmonary bypass & $0(0)$ & $3.1(2)$ & 0.284 \\
\hline Procedural mortality & $0(0)$ & $4.7(3)$ & 0.187 \\
\hline Conversion to open surgery & $2.8(1)$ & $1.6(1)$ & \\
\hline Access-related complications & $13.9(5)$ & $14.3(9)$ & 0.866 \\
\hline \multicolumn{4}{|l|}{ Bleeding } \\
\hline Amount & $468(180-790)$ & $304(129-547)$ & 0.169 \\
\hline Major & $17.6(6)$ & $6.3(4)$ & \\
\hline Life-threatening & $5.9(2)$ & $4.7(3)$ & 0.131 \\
\hline
\end{tabular}

CPR: cardiopulmonary resuscitation; IABP: intra-aortic balloon pump.

Values are given as percentage (number of patients), or mean \pm standard deviation, or median and interquartile range.

aTechnical success is defined as successful vascular access, only one valve implanted in proper anatomical location and the absence of severe aortic regurgitation or mean aortic valve gradient $>20 \mathrm{mmHg}$.

bPeriprocedural hemodynamic collapse is defined as patient requiring CPR, IABP, and/or cardiopulmonary bypass for hemodynamic management during the implantation.

TABLE 3

Postoperative complications at 30 days.

\begin{tabular}{lccc}
\hline Variable & Transaortic (TAo) & Transapical (TA) & p value \\
\hline Mortality & $8.6(3)$ & $10.9(7)$ & 0.876 \\
Safety endpoint & $11.1(4)$ & $14.1(9)$ & 0.674 \\
Myocardial infarction & $0(0)$ & $0(0)$ & \\
Stroke & & & \\
$\quad$ Minor & $5.6(2)$ & $0(0)$ & \\
$\quad$ Major & $0(0)$ & $0(0)$ & \\
Renal failure & & & \\
$\quad$ Stage 1 & $13.3(4)$ & $5.5(4)$ & \\
Stage 2 & $6.7(2)$ & $1.9(1)$ & \\
$\quad$ Stage 3 & $0(0)$ & $5.4(3)$ & \\
Permanent pacemaker & $16.7(5)$ & $1(0-2)$ & 0.116 \\
Intensive care unit stay (days) & $1(0-2)$ & $7(6-10)$ & 0.085 \\
Hospital stay (days) & $6(4-6.8)$ & 0.018 \\
\hline
\end{tabular}

Values are given as percentage (number of patients), or mean \pm standard deviation, or median and interquartile range.

\section{DISCUSSION}

In most institutes, the TA-TAVI has been the primary alternative approach for patients unsuitable for the TF. The TA approach is, however, associated with more acute complications and inferior survival than the TF (3-5). The inferior outcome of the TA is mainly caused by patient-related factors, as a more morbid patient cohort is typically selected for the TA. This might not be the only explanation as some studies have found the TA access to be an independent predictor of worse survival $(4,10)$.

The novel TAo approach might have some advantages compared to the TA. As a more familiar access to the heart surgeons, the TAo offers better bleeding control strategy, even in the worst-case scenarios of tissue disruption. Unlike the apex of the heart, well-proven substitutes for the ascending aorta are available $(6,7)$. 
TABLE 4

Echocardiographic data at 3 months $(n=73)$.

\begin{tabular}{|c|c|c|c|}
\hline Variable & Transaortic (TAo) & Transapical (TA) & $\mathrm{p}$ value \\
\hline \multicolumn{4}{|c|}{ Aortic transvalvular gradient } \\
\hline Mean $(\mathrm{mmHg})$ & $9.8 \pm 4.2$ & $8.3 \pm 2.9$ & 0.175 \\
\hline Peak (mmHg) & $14.9 \pm 6.2$ & $16.0 \pm 4.9$ & 0.442 \\
\hline LVEF & $53.0 \pm 12.6$ & $56.5 \pm 7.8$ & 0.203 \\
\hline \multicolumn{4}{|c|}{ Intravalvular aortic regurgitation } \\
\hline 0 (none-trace) & $96.3(26)$ & $76.1(35)$ & \\
\hline $1+($ mild $)$ & $3.7(1)$ & $23.9(11)$ & \\
\hline $2+($ moderate $)$ & $0(0)$ & $0(0)$ & \\
\hline $3+$ (severe) & $0(0)$ & $0(0)$ & 0.025 \\
\hline \multicolumn{4}{|c|}{ Paravalvular leakage } \\
\hline 0 (none-trace) & $51.9(14)$ & $32.6(15)$ & \\
\hline $1+($ mild $)$ & $48.1(13)$ & $60.9(28)$ & \\
\hline $2+($ moderate $)$ & $0(0)$ & $6.5(3)$ & \\
\hline $3+$ (severe) & $0(0)$ & $0(0)$ & 0.147 \\
\hline
\end{tabular}

LVEF: left ventricular ejection fraction.

Values are given as percentage (number of patients) or mean \pm standard deviation.

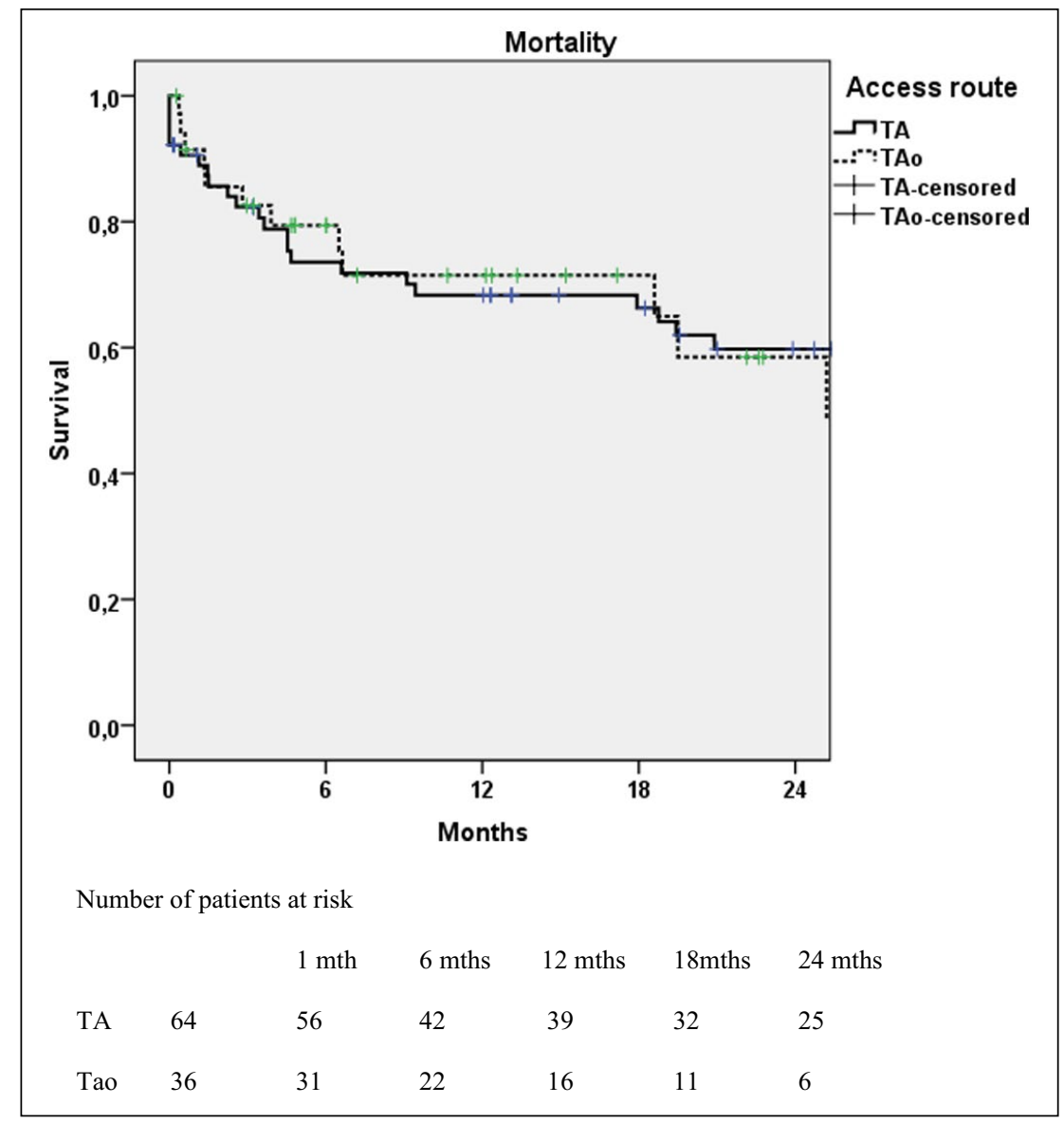

Fig. 1. The postoperative survival for the TAo and TA groups.

The TA approach directly involves myocardium of the left ventricle. It is reported that some of the TA patients develop a new myocardial hypokinesia or akinesia, and in rare cases, false aneurysm formation has been observed $(11,12)$. Furthermore, in the TA approach, the delivery system is occupying space in the left ventricle, thereby decreasing the effective ejection fraction. With a retrograde approach, this is obviated, probably translating to a hemodynamically more stable and controlled procedure. Our findings support 
TABLE 5

Causes of death.

\begin{tabular}{|c|c|c|}
\hline & Transaortic (TAo) & Transapical (TA) \\
\hline \multicolumn{3}{|l|}{ Intraprocedural } \\
\hline Aortic root rupture & & 1 \\
\hline Hemodynamic collapse & & 1 \\
\hline Severe AR & & 1 \\
\hline \multicolumn{3}{|l|}{ Within 30 days } \\
\hline Arrhythmia & & 1 \\
\hline \multicolumn{3}{|l|}{ Bleeding } \\
\hline Access-related & & 1 \\
\hline Retroperitoneal & 1 & \\
\hline Late valve migration & & 1 \\
\hline Pneumonia & 1 & \\
\hline Suffocation & 1 & \\
\hline \multicolumn{3}{|l|}{ Within 1 year } \\
\hline Cancer & 1 & \\
\hline Heart failure & 2 & 1 \\
\hline Pneumonia & 1 & 1 \\
\hline Renal failure & & 1 \\
\hline Sepsis & 1 & \\
\hline Ruptured aortic aneurysm & & 1 \\
\hline Unknown & 1 & 3 \\
\hline
\end{tabular}

AR: aortic regurgitation.

these considerations. Although the TAo group had more patients with decreased left ventricular function, we experienced significantly less difficulties in the hemodynamic management of TAo patients. Only one patient $(2.8 \%)$ needed resuscitation or mechanical circulatory support compared to $18.8 \%$ in the TA group. A total of five patients were lost intraprocedurally or within the first $24 \mathrm{~h}$, all in the TA group. However, only one of those was directly access-related due to apical bleeding that was not timely recognized.

The only major technical contraindications for the use of TAo is the inability to have a safe entry into the chest or a true porcelain aorta to a degree of not having a soft spot for aortic puncture with a minimal clearance of $5 \mathrm{~cm}$ from the aortic annulus (7). With a careful preoperative planning, successful valve implantation was achieved in all our TAo procedures, and these also included a significant proportion of patients with previous coronary artery bypass (CABG) surgery (33\% of the TAo group) and calcified ascending aortas. Similar results of successful valve implantation have been published previously, referring to the technical feasibility of this method $(6-8,13,14)$. Of note, in the TAo group larger valve sizes were implanted than in the TA counterparts in our study. This was not an access-dependent matter but rather reflects the later start of the TAo approach, when the MDCT was the primary imaging tool for aortic root analysis and valve sizing in our institution. Due to our positive experience with the TAo approach, we have moved to offer the TAo as the first alternative access for the SAPIEN valve and reserving the TA access only to those who technically can undergo neither the TF nor the TAo procedure.

As of now, it is unproven whether the potential benefits of TAo-TAVI could be translated into a better clinical outcome. There is only two small studies published where TA and TAo approaches with the SAPIEN valve are compared $(7,8)$. The first US experience reported $14 \%$ mortality in both groups at 30 days (8). In another study, mortality was $7.7 \%$ in TA group and $11.8 \%$ in TAo group at 30 days (7). Despite a higher risk patient cohort in the TAo group, our results compare favorably to these previous studies, with equal outcome in terms of mortality and combined safety endpoint in both the groups at 30 days. In contrast to Lardizabal et al. (8), we did not observe any difference in the postoperative bleeding or the ICU stay between the two groups. During the 3-month TTE follow-up (FU), there was no major intravalvular regurgitation or paravalvular leakage observed in either groups in our study. Aortic transvalvular gradients were also acceptable in both groups. These findings are encouraging considering the longterm patency of the implanted valves. In summary, our procedural success and immediate and short-term results using the TAo approach were comparable to the TA, supporting our present TAVI strategy.

Long-term survival data where TA and TAo approaches are compared are equally scarce, as there is only one study dealing with longer than 30-day data. Bapat et al. (7) reported that with a limited number of patients overall mortality rates were $28.6 \%$ for TA group at mean FU time of 425 days and $23.5 \%$ for TAo group at mean FU time of 162 days (NS). In our study, the respective 1-year survival rates for the TA and TAo groups were $68.3 \%$ and $71.5 \%$. No difference in survival between the groups was observed up to 2 years of FU. The significant mid-term mortality reflects the extreme-risk nature of patients who are selected for the alternative access TAVI. This signals a clear need for even more thorough patient screening in order to better identify those patients who are capable of achieving long-term health benefits of TAVI, irrespective of the access method chosen. 
This study has multiple obvious limitations that are inherent to a retrospective and a non-randomized single-center experience with a limited sample size. First, this study cannot be regarded as directly comparative due to individual patient selection between the groups. Second, the FU time of the patients is still quite limited. Third, the original alternative access learning curve was with the TA patients. Thus, a negative effect of the TA group results by the learning curve cannot be excluded. We did not, however, observe a trend toward an improved safety profile with increasing operator experience for the TA approach. There are studies implicating a steep learning curve for the TA with an improvement in the outcome first after 150 procedures (15). A large-scale, randomized trial is clearly warranted, but the authors are not aware of any plans of such being executed in the foreseeable future.

In conclusion, we demonstrate the technical feasibility of the TAo approach. It can be considered as an acceptable choice to TA approach. Despite a higher risk patient cohort in the TÁo group, less intraprocedural complications and similar early outcome were observed. According to our experience, the TAo approach can be instituted as a second choice for patients with prohibitive iliac-femoral anatomy for TF access. Most importantly, it gives one more access option facilitating an individualized tailoring of the transcatheter heart valve implantation for this very challenging patient population.

\section{DECLARATION OF CONFLICT OF INTERESTS}

Dr Laine is proctor for Edwards Lifesciences and Boston Scientific and Dr Ihlberg is proctor for Edwards Lifesciences. Other authors declared no potential conflicts of interest.

\section{FUNDING}

This research received no specific grant from any funding agency in the public, commercial, or not-for-profit sectors.

\section{REFERENCES}

1. Leon MB, Smith CR, Mack M, et al: Transcatheter aortic-valve implantation for aortic stenosis in patients who cannot undergo surgery. N Engl J Med 2010;363(17):1597-1607.
2. Smith CR, Leon MB, Mack MJ, et al: Transcatheter versus surgical aortic-valve replacement in high-risk patients. N Engl J Med 2011;364(23):2187-2198.

3. Bleiziffer S, Piazza N, Mazzitelli D, et al: Apical-access-related complications associated with trans-catheter aortic valve implantation. Eur J Cardiothorac Surg 2011;40(2):469-474.

4. Gilard M, Eltchaninoff H, Lung B, et al: Registry of transcatheter aortic-valve implantation in high-risk patients. N Engl J Med 2012;366(18):1705-1715.

5. Van der Boon RM, Marcheix B, Tchetche D, et al: Transapical versus transfemoral aortic valve implantation: a multicenter collaborative study. Ann Thorac Surg 2014;97(1):22-28.

6. Latsios G, Gerckens U, Grube E: Transaortic transcatheter aortic valve implantation: a novel approach for the truly "no-access option" patients. Catheter Cardiovasc Interv 2010;75(7):11291136.

7. Bapat V, Khawaja MZ, Attia R, et al: Transaortic transcatheter aortic valve implantation using Edwards Sapien valve: a novel approach. Catheter Cardiovasc Interv 2012;79(5):733-740.

8. Lardizabal JA, O'Neill BP, Desai HV, et al: The transaortic approach for transcatheter aortic valve replacement: initial clinical experience in the United States. J Am Coll Cardiol 2013;61(23):2341-2345.

9. Leon MB, Piazza N, Nikolsky E, et al: Standardized endpoint definitions for transcatheter aortic valve implantation clinical trials: a consensus report from the Valve Academic Research Consortium. J Am Coll Cardiol 2011;57:253-269.

10. Al-Attar N, Ghodbane W, Himbert D, et al: Unexpected complications of transapical aortic valve implantation. Ann Thorac Surg 2009;88(1):90-94.

11. Meyer CG, Frick M, Lotfi S, et al: Regional left ventricular function after transapical vs. transfemoral transcatheter aortic valve implantation analysed by cardiac magnetic resonance feature tracking. Eur Heart J Cardiovasc Imaging 2014;15(10):1168-1176.

12. Al-Attar N, Raffoul R, Himbert D, et al: False aneurysm after transapical aortic valve implantation. J Thorac Cardiovasc Surg 2009;137(1):e21-e22.

13. Amrane $\mathrm{H}$, Porta $\mathrm{F}$, van Boven AJ, et al: Transcatheter aortic valve implantation using a direct aortic approach: a singlecentre Heart Team experience. Interact Cardiovasc Thorac Surg 2014;19:777-781.

14. Hayashida K, Romano M, Lefèvre $T$, et al: The transaortic approach for transcatheter aortic valve implantation: a valid alternative to the transapical access in patients with no peripheral vascular option. A single center experience. Eur J Cardiothorac Surg 2013;44(4):692-700.

15. Kempfert J, Rastan A, Holzhey D, et al: Transapical aortic valve implantation: analysis of risk factors and learning experience in 299 patients. Circulation 2011;124(11 Suppl.):S124-S129.

Received: January 13, 2015

Accepted: January 27, 2015 